\title{
Correction to: Characterization of $n$-rectifiability in terms of Jones' square function: part I
}

\section{Xavier Tolsa ${ }^{1,2}$}

Published online: 10 January 2019

○ Springer-Verlag GmbH Germany, part of Springer Nature 2019

\section{Correction to: Calc. Var. (2015) 54:3643-3665 https://doi.org/10.1007/s00526-015-0917-z}

ABSTRACT. In the author's paper "Characterization of n-rectifiability in terms of Jones' square function: Part I", Calc. Var. PDE. (2015), no. 4, 3643-3665, there is a gap in the proof of Main Lemma 2.1. In this note, this gap is corrected.

\section{Introduction}

In [2], it is shown that if $\mu$ is an $n$-rectifiable measure in $\mathbb{R}^{d}$, then

$$
\int_{0}^{\infty} \beta_{2, \mu}^{n}(x, r)^{2} \frac{d r}{r}<\infty \text { for } \mu \text {-a.e. } x \in \mathbb{R}^{d} .
$$

(for the notation, see the aforementioned paper). An essential step for the proof is the following result (Main Lemma 2.1 in [2]):

Main Lemma Let $\mu$ be a finite Borel measure on $\mathbb{R}^{d}$ and let $\Gamma \subset \mathbb{R}^{d}$ be an $n$-dimensional Lipschitz graph in $\mathbb{R}^{d}$. Then

$$
\int_{0}^{\infty} \alpha_{\mu}^{n}(x, r)^{2} \frac{d r}{r}<\infty \text { for } \mathcal{H}^{n} \text {-a.e. } x \in \Gamma \text {. }
$$

The proof of this result in [2] has a gap because of an incorrect statement just above (2.22) in that paper. In this note, this gap is corrected. The new arguments are along the same lines as in [2], but they require many changes of technical type.

Supported by 2017-SGR-0395 (Catalonia) and MTM-2016-77635-P (MICINN, Spain).

The original article can be found online at https://doi.org/10.1007/s00526-015-0917-z.

$\triangle$ Xavier Tolsa

xtolsa@mat.uab.cat

1 ICREA, Passeig Lluís Companys 23, 08010 Barcelona, Catalonia, Spain

2 Departament de Matemàtiques, and BGSMath, Universitat Autònoma de Barcelona, 08193 Bellaterra, Barcelona, Catalonia, Spain 


\section{The proof}

\subsection{Preliminaries}

Given two finite Borel measures $\sigma, \mu$ on $\mathbb{R}^{d}$ and a closed ball $B \subset \mathbb{R}^{d}$, we set

$$
\operatorname{dist}_{B}(\sigma, \mu):=\sup \left\{\left|\int f d \sigma-\int f d \mu\right|: \operatorname{Lip}(f) \leq 1, \operatorname{supp}(f) \subset B\right\},
$$

where $\operatorname{Lip}(f)$ stands for the Lipschitz constant of $f$. We also set

$$
\alpha_{\mu}^{n}(B)=\frac{1}{r(B)^{n+1}} \inf _{a \geq 0, L} \operatorname{dist}_{B}\left(\mu, a \mathcal{H}_{L}^{n}\right),
$$

where the infimum is taken over all the constants $a \geq 0$ and all the $n$-planes $L$ which intersect $B$. If the $n$-plane $L$ is fixed, we write

$$
\alpha_{\mu, L}^{n}(B)=\frac{1}{r(B)^{n+1}} \inf _{a \geq 0} \operatorname{dist}_{B}\left(\mu, a \mathcal{H}_{L}^{n}\right) .
$$

We will drop the exponent $n$, and we will write $\alpha_{\mu}(x, r)$ and $\alpha_{\mu, L}(x, r)$ instead of $\alpha_{\mu}^{n}(\bar{B}(x, r))$ and $\alpha_{\mu, L}^{n}(\bar{B}(x, r))$ to shorten the notation.

\subsection{The case when $\mu$ is supported on $\Gamma$}

We will prove first the following partial result, which can be deduced by stopping time arguments analogous to some of the ones in [2]. However, for completeness, we show here the detailed proof.

Lemma 2.1 Let $\Gamma \subset \mathbb{R}^{d}$ be an n-dimensional Lipschitz, graph in $\mathbb{R}^{d}$, and let $\mu$ be a finite Borel measure supported on $\Gamma$. Then

$$
\int_{0}^{\infty} \alpha_{\mu}(x, r)^{2} \frac{d r}{r}<\infty \text { for } \mathcal{H}^{n} \text {-a.e. } x \in \Gamma .
$$

To prove the preceding result, we need to introduce the following " $\Gamma$-cubes" associated with $\Gamma$. We assume that $\Gamma$ equals the graph of a Lipschitz function $A: \mathbb{R}^{n} \rightarrow \mathbb{R}^{d-n}$. We say that $Q \subset \Gamma$ is a $\Gamma$-cube if it is a subset of the form $Q=\Gamma \cap\left(Q_{0} \times \mathbb{R}^{d-n}\right)$, where $Q_{0} \subset \mathbb{R}^{n}$ is an $n$-dimensional cube. We denote $\ell(Q):=\ell\left(Q_{0}\right)$. We say that $Q$ is a dyadic $\Gamma$-cube if $Q_{0}$ is a dyadic cube. The center of $Q$ is the point $x_{Q}=\left(x_{Q_{0}}, A\left(x_{Q_{0}}\right)\right)$, where $x_{Q_{0}}$ is the center of $Q_{0}$. The collection of dyadic $\Gamma$-cubes $Q$ with $\ell(Q)=2^{-j}$ is denoted by $\mathcal{D}_{\Gamma, j}$. Also, we set $\mathcal{D}_{\Gamma}=\bigcup_{j \in \mathbb{Z}} \mathcal{D}_{\Gamma, j}$ and $\mathcal{D}_{\Gamma}^{k}=\bigcup_{j \geq k} \mathcal{D}_{\Gamma, j}$.

Given a $\Gamma$-cube $Q$, we denote by $B_{Q}$ a closed ball concentric with $Q$ with $r\left(B_{Q}\right)=$ $3 \operatorname{diam}(Q)$. Note that $B_{Q}$ contains $Q$ and is centered on $\Gamma$. We set

$$
\alpha_{\mu}(Q):=\alpha_{\mu}\left(B_{Q}\right) .
$$

By standard methods, it follows that Lemma 2.1 is an immediate consequence of the following more precise result:

Lemma 2.2 Let $\Gamma$ be an $n$-dimensional Lipschitz graph in $\mathbb{R}^{d}$, let $\mu$ be a finite Borel measure supported on $\Gamma$, and let $\mathcal{D}_{\Gamma}$ be the lattice of $\Gamma$-cubes introduced above. Denoting by $L_{Q}$ the $n$-plane that minimizes $\alpha_{\mathcal{H}^{n}{ }_{\Gamma}}(Q)$, we have

$$
\sum_{Q \in \mathcal{D}_{\Gamma}: x \in Q} \alpha_{\mu, L_{Q}}(Q)^{2}<\infty \text { for } \mathcal{H}^{n} \text {-a.e. } x \in \Gamma \text {. }
$$


Proof First note that if $\mu=\left.g \mathcal{H}^{n}\right|_{\Gamma}$ with $g \in L^{\infty}\left(\left.\mathcal{H}^{n}\right|_{\Gamma}\right)$, then the conclusion of the lemma is true. Indeed, it turns out that the measure $\sigma:=\left.(1+g) \mathcal{H}^{n}\right|_{\Gamma}$ is $n$-AD-regular and thus

$$
\sum_{Q \in \mathcal{D}_{\Gamma}: x \in Q, \ell(Q) \leq 1} \alpha_{\sigma, L_{Q}}(Q)^{2}<\infty \text { for } \mathcal{H}^{n} \text {-a.e. } x \in \Gamma,
$$

by the results from [1]. Then, for any $Q \in \mathcal{D}_{\Gamma}$, we have

$$
\alpha_{\mu, L_{Q}}(Q) \leq \alpha_{\left.\mathcal{H}^{n}\right|_{\Gamma}, L_{Q}}(Q)+\alpha_{\sigma, L_{Q}}(Q)
$$

and thus

$$
\begin{aligned}
\sum_{Q \in \mathcal{D}_{\Gamma}: x \in Q, \ell(Q) \leq 1} \alpha_{\mu, L_{Q}}(Q)^{2} \lesssim & \sum_{Q \in \mathcal{D}_{\Gamma}: x \in Q, \ell(Q) \leq 1} \alpha_{\left.\mathcal{H}^{n}\right|_{\Gamma}, L_{Q}}(Q)^{2} \\
& +\sum_{Q \in \mathcal{D}_{\Gamma}: x \in Q, \ell(Q) \leq 1} \alpha_{\sigma, L_{Q}}(Q)^{2}<\infty \text { for } \mathcal{H}^{n} \text {-a.e. } x \in \Gamma,
\end{aligned}
$$

On the other hand, using just that $\mu$ is a finite measure, it follows easily that

$$
\sum_{Q \in \mathcal{D}_{\Gamma}: x \in Q, \ell(Q) \geq 1} \alpha_{\mu, L_{Q}}(Q)^{2}<\infty \quad \text { for all } x \in \Gamma,
$$

and so we have

$$
\sum_{Q \in \mathcal{D}_{\Gamma}: x \in Q} \alpha_{\mu, L_{Q}}(Q)^{2}<\infty \text { for } \mathcal{H}^{n} \text {-a.e. } x \in \Gamma .
$$

In the case when $\mu$ is an arbitrary finite Borel measure supported on $\Gamma$, we consider a suitable Calderón-Zygmund decomposition of $\mu$ with respect to $\left.\mathcal{H}^{n}\right|_{\Gamma}$. So for some big $\lambda>0$, we let $\mathcal{B}$ be the maximal family of $\Gamma$-cubes $P$ such that $\mu(P)>\lambda \mathcal{H}^{n}(P)$, and then we have

$$
\mu=\left.g \mathcal{H}^{n}\right|_{\Gamma}+v
$$

where

$$
\left.g \mathcal{H}^{n}\right|_{\Gamma}=\left.\mu\right|_{G}+\left.\sum_{P \in \mathcal{B}} \frac{\mu(P)}{\mathcal{H}^{n}(P)} \mathcal{H}^{n}\right|_{P}
$$

with $G=\mathbb{R}^{d} \backslash \bigcup_{P \in \mathcal{B}} P$, and also

$$
v=\sum_{P \in \mathcal{B}} v_{P}, \quad v_{P}=\left.\mu\right|_{P}-\left.\frac{\mu(P)}{\mathcal{H}^{n}(P)} \mathcal{H}^{n}\right|_{P} .
$$

By standard arguments, it is clear that $\|g\|_{L^{\infty}(\mu)} \lesssim \lambda$, and thus if we denote $\sigma=\left.g d \mathcal{H}^{n}\right|_{\Gamma}$, then

$$
\sum_{Q \in \mathcal{D}_{\Gamma}: x \in Q} \alpha_{\sigma, L_{Q}}(Q)^{2}<\infty \text { for } \mathcal{H}^{n} \text {-a.e. } x \in \Gamma .
$$

Let $\widetilde{G}=\Gamma \backslash \bigcup_{P \in \mathcal{B}} 3 B_{P}$, and observe that

$$
\mathcal{H}^{n}(\Gamma \backslash \widetilde{G})=\sum_{P \in \mathcal{B}} \mathcal{H}^{n}\left(3 B_{P}\right) \leq c \sum_{P \in \mathcal{B}} \mathcal{H}^{n}(P) \leq \frac{c}{\lambda} \sum_{P \in \mathcal{B}} \mu(P) \leq \frac{c}{\lambda}\|\mu\| .
$$


Since $\lambda$ can be taken arbitrarily big, to prove the lemma, it suffices to show that

$$
\sum_{Q \in \mathcal{D}_{\Gamma}: x \in Q} \alpha_{\mu, L_{Q}}(Q)^{2}<\infty \text { for } \mathcal{H}^{n} \text {-a.e. } x \in \widetilde{G}
$$

Denote by $\mathcal{G}$ the family of those $\Gamma$-cubes which are not contained in $\bigcup_{P \in \mathcal{B}} 3 B_{P}$. Notice that, for $x \in \widetilde{G}$, all the $\Gamma$-cubes in the sum in (2.2) are from $\mathcal{G}$. For a given $Q \in \mathcal{G}$, we have

$$
\alpha_{\mu, L_{Q}}(Q) \leq \alpha_{\sigma, L_{Q}}(Q)+\frac{1}{\ell(Q)^{n+1}} \sup _{f}\left|\int f d \nu\right|,
$$

where the supremum is taken over all functions $f$ which are 1-Lipschitz and supported on $B_{Q}$. To estimate the last integral on the right hand side, we write

$$
\left|\int f d v\right| \leq \sum_{P \in \mathcal{B}: P \cap B_{Q} \neq \varnothing}\left|\int f d v_{P}\right|
$$

Since $\int d v_{P}=0$ for all $P \in \mathcal{B}$, we have

$$
\left|\int f d v_{P}\right| \leq \int\left|f-f\left(x_{P}\right)\right| d\left|v_{P}\right| \lesssim \ell(P)\left\|v_{P}\right\| \lesssim \ell(P) \mu(P) .
$$

Thus,

$$
\alpha_{\mu, L_{Q}}(Q) \lesssim \alpha_{\sigma, L_{Q}}(Q)+\sum_{P \in \mathcal{B}: P \cap B_{Q} \neq \varnothing} \frac{\ell(P) \mu(P)}{\ell(Q)^{n+1}} .
$$

So, because of (2.1), to prove the lemma, it suffices to show that

$$
\sum_{Q \in \mathcal{G}: x \in Q}\left(\sum_{P \in \mathcal{B}: P \cap B_{Q} \neq \varnothing} \frac{\ell(P) \mu(P)}{\ell(Q)^{n+1}}\right)^{2}<\infty \text { for } \mathcal{H}^{n} \text {-a.e. } x \in \widetilde{G}
$$

To prove (2.3), first we take into account that if $P \in \mathcal{B}$ is such that $P \cap B_{Q} \neq \varnothing$, then

$$
r\left(B_{P}\right) \leq r\left(B_{Q}\right)
$$

because otherwise $Q \subset B_{Q} \subset 3 B_{P}$, which contradicts the fact that $Q \in \mathcal{G}$. Now, from (2.4), we deduce that $P \subset B_{P} \subset 3 B_{Q}$, and taking also into account that $\mu(P) \lesssim \lambda \ell(P)^{n}$, we get

$$
\sum_{P \in \mathcal{B}: P \cap B_{Q} \neq \varnothing} \frac{\ell(P) \mu(P)}{\ell(Q)^{n+1}} \lesssim \lambda \sum_{P \in \mathcal{B}: P \subset 3 B_{Q}} \frac{\ell(P)^{n+1}}{\ell(Q)^{n+1}} \lesssim \lambda
$$

Therefore,

$$
\sum_{Q \in \mathcal{G}: x \in Q}\left(\sum_{P \in \mathcal{B}: P \cap B_{Q} \neq \varnothing} \frac{\ell(P) \mu(P)}{\ell(Q)^{n+1}}\right)^{2} \lesssim \lambda \sum_{Q \in \mathcal{G}: x \in Q} \sum_{P \in \mathcal{B}: P \cap B_{Q} \neq \varnothing} \frac{\ell(P) \mu(P)}{\ell(Q)^{n+1}}
$$


Now we can show that the last sum is finite for $\mathcal{H}^{n}$-a.e. $x \in \widetilde{G}$ by integrating this with respect to $\left.\mathcal{H}^{n}\right|_{\Gamma}$. Then we obtain

$$
\begin{aligned}
\int_{\Gamma} \sum_{Q \in \mathcal{G}: x \in Q} \sum_{P \in \mathcal{B}: P \cap B_{Q} \neq \varnothing} \frac{\ell(P) \mu(P)}{\ell(Q)^{n+1}} d \mathcal{H}^{n}(x) & \approx \sum_{Q \in \mathcal{G}} \sum_{P \in \mathcal{B}: P \cap B_{Q} \neq \varnothing} \frac{\ell(P) \mu(P)}{\ell(Q)} \\
& =\sum_{P \in \mathcal{B}} \mu(P) \sum_{Q \in \mathcal{G}: P \cap B_{Q} \neq \varnothing} \frac{\ell(P)}{\ell(Q)} .
\end{aligned}
$$

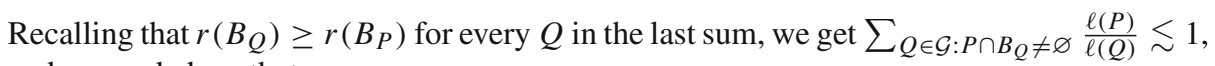
and so we deduce that

$$
\int_{\Gamma} \sum_{Q \in \mathcal{G}: x \in Q} \sum_{P \in \mathcal{B}: P \cap B_{Q} \neq \varnothing} \frac{\ell(P) \mu(P)}{\ell(Q)^{n+1}} d \mathcal{H}^{n}(x) \lesssim \sum_{P \in \mathcal{B}} \mu(P) \leq\|\mu\|,
$$

which implies (2.3) and completes the proof of the lemma.

\subsection{The approximating measure $\sigma$ for the general case}

To prove the main lemma in full generality, we consider a Whitney decomposition of $\mathbb{R}^{d} \backslash \Gamma$ as in Section 2.3 of [2]. That is, we have a family $\mathcal{W}$ of dyadic cubes in $\mathbb{R}^{d}$ with disjoint interiors such that

$$
\bigcup_{Q \in \mathcal{W}} Q=\mathbb{R}^{d} \backslash \Gamma
$$

and moreover, there are some constants $R>20$ and $D_{0} \geq 1$ such the following holds for every $Q \in \mathcal{W}$ :

(i) $10 Q \subset \mathbb{R}^{d} \backslash \Gamma$;

(ii) $R Q \cap \Gamma \neq \varnothing$;

(iii) there are at most $D_{0}$ cubes $Q^{\prime} \in \mathcal{W}$ such that $10 Q \cap 10 Q^{\prime} \neq \varnothing$. Further, for such cubes $Q^{\prime}$, we have $\ell\left(Q^{\prime}\right) \approx \ell(Q)$.

From properties (i) and (ii), it is clear that $\operatorname{dist}(Q, \Gamma) \approx \ell(Q)$. We assume that the Whitney cubes are small enough so that

$$
\operatorname{diam}(Q)<\operatorname{dist}(Q, \Gamma) .
$$

This can be achieved by replacing each cube $Q \in \mathcal{W}$ by its descendants $P \in \mathcal{D}_{k}(Q)$, for some fixed $k \geq 1$, if necessary. From (2.5), we infer that if $Q \in \mathcal{W}$ intersects some ball $B(y, r)$ with $y \in \Gamma$, then

$$
\operatorname{diam}(Q) \leq r
$$

and thus

$$
Q \subset B(y, 3 r) .
$$

For a given Borel measure $\mu$, we denote

$$
M_{n} \mu(x)=\sup _{r>0} \frac{\mu(B(x, r))}{r^{n}} .
$$

From the growth condition $\mathcal{H}^{n}(\Gamma \cap B(x, r)) \leq c r^{n}$ for all $x$ and $r>0$, it follows easily that the maximal operator $M_{n}$ is bounded from the space of finite signed Radon measures 
$M\left(\mathbb{R}^{d}\right)$ into $L^{1, \infty}\left(\left.\mathcal{H}^{n}\right|_{\Gamma}\right)$. As a consequence, for any arbitrary finite Borel measure $\mu$ in $\mathbb{R}^{d}$, $M_{n} \mu(x)<\infty$ for $\mathcal{H}^{n}$-a.e $x \in \Gamma$.

Denote by $\Pi_{\Gamma}$ the projection on $\Gamma$ given by $\Pi_{\Gamma}(x)=\left(x_{1}, \ldots, x_{n}, A\left(x_{1}, \ldots, x_{n}\right)\right)$. To each cube $Q \in \mathcal{W}$ we associate a ball $\widetilde{B}_{Q}$ with radius $r\left(\widetilde{B}_{Q}\right)=\frac{1}{4} \ell(Q)$, centered in $\widetilde{x}_{Q}:=\Pi_{\Gamma}\left(x_{Q}\right)$, where $x_{Q}$ is the center of $Q$. In particular, we have $\operatorname{dist}\left(Q, \widetilde{B}_{Q}\right) \approx \ell(Q) \approx r\left(\widetilde{B}_{Q}\right)$.

Let $\varphi: \mathbb{R}^{d} \rightarrow \mathbb{R}$ be a smooth non-negative radial function supported in $B(0,1)$ which equals 1 in $B(0,1 / 2)$. For each $Q \in \mathcal{W}$, we consider the function $\varphi_{Q}: \mathbb{R}^{d} \rightarrow \mathbb{R}$ defined by

$$
\varphi_{Q}(x)=\varphi\left(\frac{x-\widetilde{x}_{Q}}{r\left(\widetilde{B}_{Q}\right)}\right)
$$

and then we set

$$
g_{Q}(x)=\frac{\mu(Q)}{\left\|\varphi_{Q}\right\|_{L^{1}\left(\mathcal{H}^{n} \mid \Gamma\right)}} \varphi_{Q}(x),
$$

and also

$$
g(x)=\sum_{Q \in \mathcal{W}} g_{Q}(x)
$$

Notice that $\left.\int g_{Q} d \mathcal{H}^{n}\right|_{\Gamma}=\mu(Q)$ and also that

$$
\left\|g_{Q}\right\|_{\infty} \lesssim \frac{\mu(Q)}{\ell(Q)^{n}}, \quad\left\|\nabla g_{Q}\right\|_{\infty} \lesssim \frac{\mu(Q)}{\ell(Q)^{n+1}}
$$

Then we consider the following measure $\sigma$ on $\Gamma$, which approximates $\left.\mu\right|_{\Gamma^{c}}$, in a sense:

$$
\sigma=\left.g d \mathcal{H}^{n}\right|_{\Gamma}=\left.\sum_{Q \in \mathcal{W}} g_{Q} d \mathcal{H}^{n}\right|_{\Gamma}
$$

Next, for some constant $A>10$ to be chosen below, we also consider the auxiliary function

$$
G_{A}(x)=\sum_{Q \in \mathcal{W}} \frac{\mu(Q)}{\ell(Q)^{n}} \chi_{A \widetilde{B}_{Q}}
$$

Notice that, for some absolute constant $C$,

$$
g(x) \leq C G_{A}(x) \text { for all } x \in \Gamma .
$$

Also,

$$
\left\|G_{A}\right\|_{L^{1}\left(\mathcal{H}^{n} \mid \Gamma\right)} \leq C \sum_{Q \in \mathcal{W}} \frac{\mu(Q) A^{n} r\left(\widetilde{B}_{Q}\right)^{n}}{\ell(Q)^{n}} \leq C A^{n}\|\mu\|,
$$

and thus $G_{A}(x)<\infty$ for $\mathcal{H}^{n}$-a.e. $x \in \Gamma$. 


\subsection{The $\alpha_{\mu}$ coefficients of the $\Gamma$-cubes}

Lemma 2.3 Assume A big enough. Then, for each $Q \in \mathcal{D}_{\Gamma}$, we have

$$
\begin{aligned}
\alpha_{\mu}(Q) \lesssim & \alpha_{\left.\mu\right|_{\Gamma}, L_{Q}}(Q)+\alpha_{\sigma, L_{Q}}(Q)+\int_{6 B_{Q}} \frac{\operatorname{dist}(x, \Gamma)}{\ell(Q)^{n+1}} d \mu(x) \\
& +\inf _{x \in Q} G_{A}(x) \alpha_{\left.\mathcal{H}^{n}\right|_{\Gamma}}(Q)+\sum_{\substack{P \in \mathcal{W}: P \cap 2 B_{Q}=\varnothing, \widetilde{B}_{P} \cap B_{Q} \neq \varnothing}} \frac{\mu(P) \ell(Q)}{\ell(P)^{n+1}},
\end{aligned}
$$

where $L_{Q}$ is the n-plane that minimizes $\left.\alpha_{\mathcal{H}^{n}}\right|_{\Gamma}(Q)$.

Proof Note first that $\alpha_{\mu}(Q) \leq \alpha_{\left.\mu\right|_{\Gamma}, L_{Q}}(Q)+\alpha_{\left.\mu\right|_{\Gamma^{c}, L_{Q}}}(Q)$. So we just have to deal with $\alpha_{\left.\mu\right|_{\Gamma^{c}}, L_{Q}}(Q)$. To estimate this coefficient, we consider a 1-Lipschitz function $f$ supported on $B_{Q}$, and we write

$$
\left.\int f d \mu\right|_{\Gamma^{c}}=\sum_{P \in \mathcal{W}} \int f d\left(\left.\mu\right|_{P}-\left.g_{P} \mathcal{H}^{n}\right|_{\Gamma}\right)+\int f d \sigma,
$$

where $g_{P}$ is the function defined in (2.8) and $\sigma$ the measure in (2.9).

So, for any constant $a_{Q} \in \mathbb{R}$, we get

$$
\alpha_{\left.\mu\right|_{\Gamma^{c}, L_{Q}}}(Q) \leq \alpha_{\sigma, L_{Q}}(Q)+\frac{1}{\ell(Q)^{n+1}} \sup _{f}\left|\sum_{P \in \mathcal{W}} \int f d\left(\left.\mu\right|_{P}-\left.g_{P} \mathcal{H}^{n}\right|_{\Gamma}\right)+a_{Q} \int f d \mathcal{H}^{n}\right|_{L_{Q}} \mid,
$$

where the supremum is taken over all 1-Lipschitz functions supported on $B_{Q}$.

We denote by $I_{a}$ the subfamily of the cubes from $\mathcal{W}$ which intersect $2 B_{Q}$, and $I_{b}=\mathcal{W} \backslash I_{a}$, and we split the last sum above as follows:

$$
\begin{aligned}
& \left|\sum_{P \in \mathcal{W}} \int f d\left(\left.\mu\right|_{P}-\left.g_{P} \mathcal{H}^{n}\right|_{\Gamma}\right)+a_{Q} \int f d \mathcal{H}^{n}\right|_{L_{Q}} \mid \\
& \quad \leq \sum_{P \in I_{a}}\left|\int f d\left(\left.\mu\right|_{P}-\left.g_{P} \mathcal{H}^{n}\right|_{\Gamma}\right)\right|+\left|\sum_{P \in I_{b}} \int f d\left(\left.\mu\right|_{P}-\left.g_{P} \mathcal{H}^{n}\right|_{\Gamma}\right)+a_{Q} \int f d \mathcal{H}^{n}\right|_{L_{Q}} \mid \\
& \quad=: S_{a}+S_{b} .
\end{aligned}
$$
that

First we deal with the sum $S_{a}$. For each $P \in I_{a}$, since $\left.\int g_{P} d \mathcal{H}^{n}\right|_{\Gamma}=\mu(P)$, we deduce

$$
\begin{aligned}
\left|\int f d\left(\left.\mu\right|_{P}-\left.g_{P} \mathcal{H}^{n}\right|_{\Gamma}\right)\right| \leq & \left|\int_{P}\left(f(x)-f\left(x_{P}\right)\right) d \mu(x)\right| \\
& \left.+\left|\int\left(f\left(x_{P}\right)-f(x)\right) g_{P}(x) \mathcal{H}^{n}\right|_{\Gamma}(x)\right) \mid .
\end{aligned}
$$

To deal with the first integral on the right-hand side, we take into account that for $x \in P$ we have

$$
\left|f(x)-f\left(x_{P}\right)\right| \leq\|\nabla f\|_{\infty}\left|x-x_{P}\right| \leq c \ell(P) .
$$

Concerning the second integral, recall that supp $g_{P} \subset \Gamma \cap \bar{B}\left(x_{P}, c \ell(P)\right)$, and thus we also have $\left|x-x_{P}\right| \leq c \ell(P)$ in the domain of integration, so that (2.10) holds in this case too. 
Therefore,

$$
\left|\int f d\left(\left.\mu\right|_{P}-\left.g_{P} \mathcal{H}^{n}\right|_{\Gamma}\right)\right| \leq c \ell(P) \mu(P) \approx \int_{P} \operatorname{dist}(x, \Gamma) d \mu(x),
$$

where we took into account that $\operatorname{dist}(x, \Gamma) \approx \ell(P)$ for every $x \in P$. Recall that supp $f \subset B_{Q}$ and since $P \in I_{a}$, then $P \subset 6 B_{Q}$ by the argument in (2.7). Thus,

$$
S_{a}=\sum_{P \in I_{a}}\left|\int f d\left(\left.\mu\right|_{P}-\left.g_{P} \mathcal{H}^{n}\right|_{\Gamma}\right)\right| \leq c \int_{6 B_{Q}} \operatorname{dist}(x, \Gamma) d \mu(x) .
$$

Next we consider the sum $S_{b}$. For each $P \in I_{b}$, we have $P \cap 2 B_{Q}=\varnothing$, and so $\left.\int f d \mu\right|_{P}=$ 0 . Therefore,

$$
\begin{aligned}
S_{b} & =\left|\sum_{P \in I_{b}} \int f g_{P} d \mathcal{H}^{n}\right|_{\Gamma}-\left.a_{Q} \int f d \mathcal{H}^{n}\right|_{L_{Q}} \mid \\
& \leq\left|\sum_{P \in I_{b}} \int f g_{P} d\left(\left.\mathcal{H}^{n}\right|_{\Gamma}-\left.c_{Q} \mathcal{H}^{n}\right|_{L_{Q}}\right)\right|+\left.\int|f|\left|\sum_{P \in I_{b}} c_{Q} g_{P}-a_{Q}\right| d \mathcal{H}^{n}\right|_{L_{Q}} \\
& =: S_{b, 1}+S_{b, 2},
\end{aligned}
$$

where $c_{Q}$ is the constant minimizing $\alpha_{\left.\mathcal{H}^{n}\right|_{\Gamma}, L_{Q}}(Q)$. To deal with $S_{b, 1}$ we take into account that $\sum_{P \in I_{b}} f g_{P}$ is a Lipschitz function supported in $B_{Q}$, and $f g_{P}$ vanishes unless $\widetilde{B}_{P} \cap B_{Q} \neq \varnothing$. To shorten notation, we write $P \sim Q$ if $P \in I_{b}$ is such that $\widetilde{B}_{P} \cap B_{Q} \neq \varnothing$. It is easy to check that $P \sim Q$ implies that $\ell(P) \gtrsim \ell(Q)$. Then we have

$$
\begin{aligned}
\left\|\nabla\left(\sum_{P \in I_{b}} f g_{P}\right)\right\|_{\infty} & \leq \sum_{P \sim Q}\left(\left\|g_{P}\right\|_{\infty}+c \ell(Q)\left\|\nabla g_{P}\right\|_{\infty}\right) \\
& \lesssim \sum_{P \sim Q}\left(\frac{\mu(P)}{\ell(P)^{n}}+\frac{\ell(Q) \mu(P)}{\ell(P)^{n+1}}\right) \lesssim \sum_{P \sim Q} \frac{\mu(P)}{\ell(P)^{n}} .
\end{aligned}
$$

From the fact that $\ell(P) \gtrsim \ell(Q)$, it also follows that, for $A$ big enough, we have

$$
\sum_{P \sim Q} \frac{\mu(P)}{\ell(P)^{n}} \lesssim \inf _{x \in Q} G_{A}(x)
$$

Therefore,

$$
S_{b, 1} \lesssim \alpha_{\left.\mathcal{H}^{n}\right|_{\Gamma}}(Q) \ell(Q)^{n+1} \inf _{x \in Q} G_{A}(x)
$$

Finally, we turn our attention to the term $S_{b, 2}$. Choosing $a_{Q}=c_{Q} \sum_{P \sim Q} g_{P}\left(x_{Q}\right)$ and using that $\left|c_{Q}\right| \lesssim 1$, we obtain

$$
\left.S_{b, 2} \lesssim \int\left|f \sum_{P \sim Q}\left[g_{P}-g_{P}\left(x_{Q}\right)\right]\right| d \mathcal{H}^{n}\right|_{L_{Q}}
$$

Note now that, for $x \in B_{Q}$,

$$
\sum_{P \sim Q}\left|g_{P}(x)-g_{P}\left(x_{Q}\right)\right| \lesssim \sum_{P \sim Q}\left\|\nabla g_{P}\right\|_{\infty} \ell(Q) \lesssim \sum_{P \sim Q} \frac{\mu(P) \ell(Q)}{\ell(P)^{n+1}},
$$


and so, using also that $|f| \leq C \ell(Q) \chi_{B_{Q}}$,

$$
S_{b, 2} \lesssim \sum_{P \sim Q} \frac{\mu(P) \ell(Q)^{n+2}}{\ell(P)^{n+1}}
$$

Gathering all the estimates above, we obtain

$$
\begin{aligned}
& \alpha_{\left.\mu\right|_{\Gamma^{c}}, L_{Q}}(Q) \lesssim \alpha_{\sigma, L_{Q}}(Q)+\int_{6 B_{Q}} \frac{\operatorname{dist}(x, \Gamma)}{\ell(Q)^{n+1}} d \mu(x)+\inf _{x \in Q} G_{A}(x) \alpha_{\left.\mathcal{H}^{n}\right|_{\Gamma}}(Q) \\
& \quad+\sum_{P \sim Q} \frac{\mu(P) \ell(Q)}{\ell(P)^{n+1}}
\end{aligned}
$$

which completes the proof of the lemma.

\subsection{Proof of the Main Lemma}

It is immediate to check that the statement in the main lemma is equivalent to the fact that

$$
\sum_{Q \in \mathcal{D}_{\Gamma}} \alpha_{\mu}(Q)^{2} \chi_{Q}(x)<\infty \text { for } \mathcal{H}^{n} \text {-a.e. } x \in \Gamma \text {. }
$$

To prove this estimate, we intend to use Lemma 2.3. To this end, observe that, by Lemma 2.1, we know that

$$
\sum_{Q \in \mathcal{D}_{\Gamma}}\left(\alpha_{\left.\mu\right|_{\Gamma}, L_{Q}}(Q)^{2}+\alpha_{\sigma, L_{Q}}(Q)^{2}\right) \chi_{Q}(x)<\infty \text { for } \mathcal{H}^{n} \text {-a.e. } x \in \Gamma .
$$

Also, for every $x \in \Gamma$,

$\sum_{Q \in \mathcal{D}_{\Gamma}: Q \ni x} \inf _{y \in Q} G_{A}(y)^{2} \alpha_{\mathcal{H}^{n} \mid \Gamma}(Q)^{2} \leq G_{A}(x)^{2} \sum_{Q \in \mathcal{D}_{\Gamma}: Q \ni x} \alpha_{\mathcal{H}^{n} \mid \Gamma}(Q)^{2}<\infty$ for $\mathcal{H}^{n}$-a.e. $x \in \Gamma$, since $G_{A}(x)<\infty$ and $\sum_{Q \in \mathcal{D}_{\Gamma}: Q \ni x} \alpha_{\left.\mathcal{H}^{n}\right|_{\Gamma}}(Q)^{2}<\infty$ for $\mathcal{H}^{n}$-a.e. $x \in \Gamma$.

Next we show that

$$
\sum_{Q \in \mathcal{D}_{\Gamma}}\left(\sum_{\substack{P \in \mathcal{W}: P \cap 2 B_{Q}=\varnothing, \widetilde{B}_{P} \cap B_{Q} \neq \varnothing}} \frac{\mu(P) \ell(Q)}{\ell(P)^{n+1}}\right)^{2} \chi_{Q}(x)<\infty \text { for } \mathcal{H}^{n} \text {-a.e. } x \in \Gamma .
$$

To this end, note that, as in (2.12),

$$
\sum_{\substack{P \in \mathcal{W}: P \cap 2 B_{Q}=\varnothing, \widetilde{B}_{P} \cap B_{Q} \neq \varnothing}} \frac{\mu(P) \ell(Q)}{\ell(P)^{n+1}} \chi_{Q}(x) \lesssim \sum_{\substack{P \in \mathcal{W}: P \cap 2 B_{Q}=\varnothing, \widetilde{B}_{P} \cap B_{Q} \neq \varnothing}} \frac{\mu(P)}{\ell(P)^{n}} \chi_{Q}(x) \lesssim G_{A}(x) .
$$

Hence,

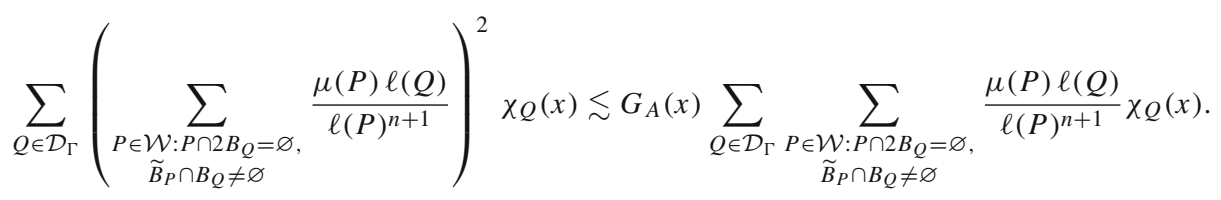


Thus to prove (2.14), it suffices to show that

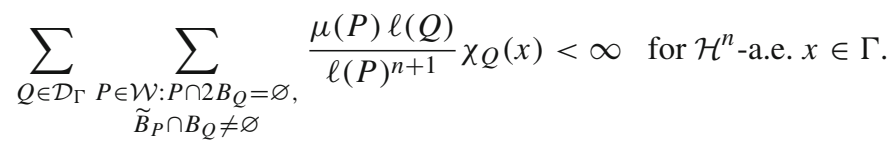

By Fubini, we have

$$
\begin{aligned}
& \left.\int \sum_{Q \in \mathcal{D}_{\Gamma}} \sum_{\substack{P \in \mathcal{W}: P \cap 2 B_{Q}=\varnothing, \widetilde{B}_{P} \cap B_{Q} \neq \varnothing}} \frac{\mu(P) \ell(Q)}{\ell(P)^{n+1}} \chi_{Q}(x) d \mathcal{H}^{n}\right|_{\Gamma}(x) \\
& \approx \sum_{Q \in \mathcal{D}_{\Gamma}} \sum_{\substack{P \in \mathcal{W}: P \cap 2 B_{Q}=\varnothing, \widetilde{B}_{P} \cap B_{Q} \neq \varnothing}} \frac{\mu(P) \ell(Q)}{\ell(P)^{n+1}} \ell(Q)^{n} \\
& \approx \sum_{P \in \mathcal{W}} \mu(P) \sum_{\substack{Q \in \mathcal{D}_{\Gamma}: P \cap 2 B_{Q}=\varnothing, \widetilde{B}_{P} \cap B_{Q} \neq \varnothing}} \frac{\ell(Q)^{n+1}}{\ell(P)^{n+1}} \lesssim \sum_{P \in \mathcal{W}} \mu(P)=\|\mu\|,
\end{aligned}
$$

where in the last inequality we took into account that the $\Gamma$-cubes $Q \in \mathcal{D}_{\Gamma}$ such that $P \cap$ $2 B_{Q}=\varnothing$ and $\widetilde{B}_{P} \cap B_{Q} \neq \varnothing$ satisfy $Q \subset c \widetilde{B}_{P}$ for some $c>1$, because $\ell(Q) \lesssim \ell(P)$. So (2.15) is proved.

Finally, to complete the proof of this lemma, by Lemma 2.3, it remains to show that

$$
\sum_{Q \in \mathcal{D}_{\Gamma}}\left(\int_{6 B_{Q}} \frac{\operatorname{dist}(y, \Gamma)}{\ell(Q)^{n+1}} d \mu(y)\right)^{2} \chi_{Q}(x)<\infty \text { for } \mathcal{H}^{n} \text {-a.e. } x \in \Gamma \text {. }
$$

The arguments for this are almost the same as the ones in [2]. We repeat them here for the reader's convenience. By Cauchy-Schwarz, we get

$$
\left(\int_{6 B_{Q}} \frac{\operatorname{dist}(y, \Gamma)}{\ell(Q)^{n+1}} d \mu(y)\right)^{2} \leq \mu\left(6 B_{Q}\right) \int_{6 B_{Q}}\left(\frac{\operatorname{dist}(y, \Gamma)}{\ell(Q)^{n+1}}\right)^{2} d \mu(y) .
$$

Since $\frac{\mu\left(6 B_{Q}\right)}{\ell(Q)^{n}} \lesssim M_{n} \mu(x)$ for all $x \in Q$, and $M_{n} \mu(x)<\infty$ for $\mathcal{H}^{n}$-a.e. $x \in \Gamma$, it turns that, to prove (2.16), it suffices to show that

$$
\sum_{Q \in \mathcal{D}_{\Gamma}} \int_{6 B_{Q}} \frac{\operatorname{dist}(y, \Gamma)^{2}}{\ell(Q)^{n+2}} d \mu(y) \chi_{Q}(x)<\infty \text { for } \mathcal{H}^{n} \text {-a.e. } x \in \Gamma .
$$

The integral of the left-hand side of (2.17) with respect to $\left.\mathcal{H}^{n}\right|_{\Gamma}$ does not exceed

$$
c \sum_{Q \in \mathcal{D}_{\Gamma}} \int_{6 B_{Q}} \frac{\operatorname{dist}(y, \Gamma)^{2}}{\ell(Q)^{n+2}} d \mu(y) \ell(Q)^{n}=c \sum_{Q \in \mathcal{D}_{\Gamma}} \int_{6 B_{Q}} \frac{\operatorname{dist}(y, \Gamma)^{2}}{\ell(Q)^{2}} d \mu(y) .
$$

By Fubini, this equals

$$
c \int \operatorname{dist}(y, \Gamma)^{2} \sum_{Q \in \mathcal{D}_{\Gamma}} \chi_{6 B_{Q}}(y) \frac{1}{\ell(Q)^{2}} d \mu(y) .
$$

Notice now that

$$
\sum_{Q \in \mathcal{D}_{\Gamma}} \chi_{6 B_{Q}}(y) \frac{1}{\ell(Q)^{2}}=\sum_{Q \in \mathcal{D}_{\Gamma}: y \in 6 B_{Q}} \frac{1}{\ell(Q)^{2}} \lesssim \frac{1}{\operatorname{dist}(y, \Gamma)^{2}},
$$


because the condition $y \in 6 B_{Q}$ implies that $\operatorname{dist}(y, \Gamma) \leq r\left(B_{Q}\right) \approx \ell(Q)$. Thus,

$$
\sum_{Q \in \mathcal{D}_{\Gamma}} \int_{6 B_{Q}} \frac{\operatorname{dist}(y, \Gamma)^{2}}{\ell(Q)^{2}} d \mu(y) \lesssim \int \frac{\operatorname{dist}(y, \Gamma)^{2}}{\operatorname{dist}(y, \Gamma)^{2}} d \mu(y)=\|\mu\| .
$$

Hence, (2.16) follows and we are done.

\section{References}

1. Tolsa, X.: Uniform rectifiability, Calderón-Zygmund operators with odd kernel, and quasiorthogonality. Proc. Lond. Math. Soc. 98(2), 393-426 (2009)

2. Tolsa, X.: Characterization of n-rectifiability in terms of Jones' square function: part I. Calc. Var. PDE 25(4), 3643-3665 (2015)

Publisher's Note Springer Nature remains neutral with regard to jurisdictional claims in published maps and institutional affiliations. 